\title{
Shared Memories: A Trail-based Coordination Server for Robot Teams
}

\author{
G. Roussos, D. Papadogkonas, J. Taylor, D. Airantzis, M.Levene and M. Zoumboulakis \\ School of Computer Science and Information Systems \\ Birkbeck College, University of London, London WC1E 7HX, U.K. \\ Email: \{dikaios,jenson,dimitrios,mz,mark,gr\}@dcs.bbk.ac.uk
}

\begin{abstract}
Robust, dependable and concise coordination between members of a robot team is a critical ingredient of any such collective activity. Depending on the availability and the characteristics of the particular communication infrastructure, coordination mechanisms can take varied forms, leading to distinct system behaviors. In this paper, we consider the case of robot teams operating within relatively sparse wireless sensor network deployments. We introduce Shared Memories, a trailbased coordination engine, that analyzes interaction patterns between participating team members and sensor network nodes capable to discover significant aggregate patterns, which are made available to the team. To this end, we propose a model for the representation of captured interactions and their sensory context developed as a probabilistic grammar, as well as associated metrics used to rank trails and quantify their significance. Such trails are used as the basis for coordinated operation in team tasks and are made available by the engine to all team members. Our implementation deploys ad-hoc wireless local networking capability available through surrogate devices to commodity robots and RFID proximity sensors. We report on the performance of this system in experiments conducted in a laboratory environment, which highlight the advantages and limitations of our approach.
\end{abstract}

\section{INTRODUCTION}

The traditional robotics approach in capturing environmental information is one of self-sufficiency, that is each agent employs its own sensing capability to make sense of the environment around it and make task-related and navigation decisions. This mode of operation is challenged by two relatively recent developments: the wider availability of wireless adhoc networks which offer advanced networking opportunities; and the rapid proliferation of wireless sensor networks which establish a richer source of environmental information that can be employed to improve operational effectiveness. This recent shift in focus inevitably places greater importance in teams rather than individuals and the use of extended sensing capabilities as the enabler of new off-device functionalities. A critical challenge in capitalizing on these new developments is the management of the collective experience acquired in such a way, and the extraction of useful and usable knowledge.

In this paper we propose a particular approach in organizing the collective information harvested by a robotic team and effective ways of providing cues for coordination employing a trail-based approach. We show within a simple feasibility study of this proposal how this approach can provide the foundation for effective coordinated operation. In addition to the use of trail records as the principle data primitive, our case study implements a simple centralized coordination server with which team members communicate in an ad-hoc basis whenever wireless connectivity becomes available.

The remainder of this paper is organized as follows: fist, we present the functional characteristics of mixed sensor network and robotic environments and proceed to describe how a trail based abstraction and associated data models and algorithms meet well its requirements. We then proceed to describe an efficient implementation of this model in terms of data structures and software components. Then we discuss a particular case study that has been developed to provide early validation of this proposal. We conclude by reporting on our experiences and future work and by raising some common themes with research carried out by other groups.

\section{MIXED PERVASIVE-ROBOTIC ENVIRONMENTS}

The context of this work lies at the intersection of pervasive computing, in particular wireless sensor networks, and robotics. We anticipate a situation where the environment is instrumented with wireless sensor and actuator networks in configurations of varying density and with varying capabilities, including location and proximity, environmental and chemical sensors, magnetic and possibly visual components. Such deeply embedded systems follow a unique modusoperandi in that they may be only intermittently connected within frequently disconnected environments. In such systems, islands of connected devices appear, disappear, and reconfigure dynamically. As a result, the network is extremely dynamic and constructed ad-hoc, its formation frequently resulting by the evolving contacts among fixed and mobile devices, and among connected constellations of devices. In this view, internet connectivity is merely a specific connectivity opportunity.

Within this context, robotic agents with the capability to communicate with the sensor network provide extended node mobility opportunities that can offer considerable advantages for network robustness and extended connectivity. Without doubt, mobility is a core ingredient for effective wireless sensor systems and can guarantee routing towards external systems and thus comprehensive networking capacity.

While such ad-hoc connectivity is undoubtedly a valuable feature of such systems, even more important are the advantages offered by the collaborative information processing opportunities supported by such integrated wireless sensor network and robotic systems. On the one hand, robotic teams 
can relay information between sensor nodes that have become disconnected to allow for collaborative and cluster based processing and can also temporarily allow the use of their superior computational resources that would support the implementation of more complex mechanisms and the execution of more demanding tasks than what would normally be possible by the highly restricted sensor nodes. A feature of this technology mix that is more relevant to the discussions of this paper is that interaction between sensor nodes and robots can provide a powerful means for advanced coordinated operation.

Such co-operation can take many forms and in this paper we propose a particular approach built on trails as the data primitive and a centralized approach to coordination. In particular, we introduce a universal model for the capture of such interactions and their representation in such a way that the complete information captured by the robotic team in this way is organized in such a way that common patterns can be quickly identified and individualized assistance to particular team members can be tailored in a highly efficient manner that can cope with the large amount of collected information.

\section{TRAIL-BASED COORDINATION}

The core ingredient of our approach is the use of trails as the principal data processing primitive for analysis and prediction. We define a trail of an individual robotic agent as the sequence of recorded interactions between itself and the nodes of a wireless sensor network. Trails contain patterns of actions and they can be used for the provision of different services, spatial analysis or navigational assistance. A downside of trail analysis is that it requires considerable storage and computational resources to discover such patterns. Moreover no single method exists that identifies significant trails based on different metrics related to a particular application. Here, we introduce a trail based analysis approach, an associated model for the representation of trails and trail aggregates, and suitable data structures for efficient storage, filtering and retrieval.

To identify specific types of interactions we introduce the notion of the landmark as the position of significant object in a mixed pervasive-robotic landscape. Interactions recorded in trails represent communications between a robot and the node located at a specific landmark which are associated with both spatial and semantic attributes induced by the embedding of sensor networks in physical space.

The choice of trails as our representation of choice is not coincidental. Indeed, trail records have been used as the basis for coordination between humans for centuries in different forms. Navigation trails for example provide route information and record information about paths to specific destinations. Aggregating multiple trails acquired over time across a particular environment is the technique humans often use to develop complete maps of a particular landscape and subsequently assist navigation, especially in the context of exploration [5]. Oral trails are also quite common in human coordination and are best represented as narratives which are replayed and recast repeatedly to incorporate new knowledge [9].

More relevant to the current discussion, trails have been used with great success in assisting coordination of information seeking activities on the Web [2]. Our work is intimately related to this approach and proposes extensions to this model of collective experience aggregation to cater for the distinct requirements of environments that mix wireless sensor networks and robotics. Here we propose a model that organizes the complete information captured by all members of a robotic team into a unified representation and propose a number of metrics that can be used to identify the most important of these trails or trails that are simply of value when carrying out a particular task. Such so-called significant trails are disseminated and used to provide guidance and assistance in a variety of coordinated tasks.

\section{Shared Memories CoORdination Engine}

To construct the Shared Memories coordinator we introduce the interaction network that is, a graph where nodes represent sensor network nodes. In the interaction network two nodes are connected when there is a corresponding interaction record which indicates that the two landmarks have been visited in sequence by at least one robot. These (directed) links between landmarks are weighted with different usage measures for example, frequency of visitation. Higher order weights may also be employed for example, the compound probability that a link will be followed given that the robot has arrived to this node following a specific path of fixed length within the network.

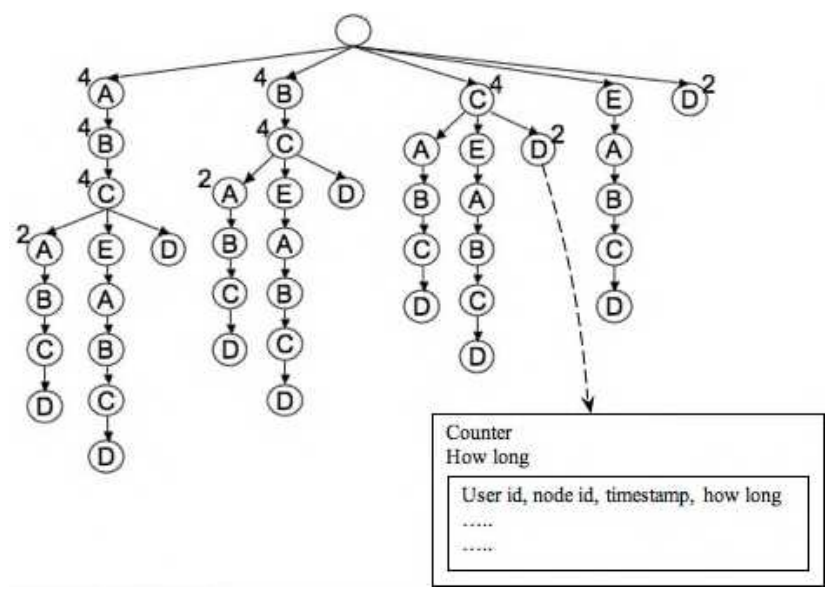

Fig. 1. Suffix tree containing two trails, namely $A B C A B C D$ and $A B C E-$ $A B C D$. Each letter represents a wireless sensor nodes with which at least one robot has interacted with. Each node is also associated with additional metadata used to answer and rank significant trail queries. trails with an example of the information associated with a node.

Each graph node also holds additional metadata that relate to the semantic attributes of the sensor node in consideration and largely depend on its capabilities. Node meta-data also record different aspects of the interaction with robots for example, the distance between the robot and the sensor node during their interactive session, the length of time the interaction lasted or 
the orientation of the robot towards the wireless sensor node. It should be noted that not all sensor systems can provide this information nor does it make sense for all types of landmarks. In any case, calculating the weights from the raw system logs requires considerable effort and poses several challenges in reconciling the records. For example different sub-systems may utilize different $\log$ file formats, may record different aspects of the interaction, may not be synchronized, may have different time recording granularities or have different schemes to identify landmarks.

Trails are represented as a sequence of nodes within the graph. For efficiency, the graph is stored as a probabilistic suffix tree [11] enhanced to encapsulate different information and metrics regarding each interaction [10]. An example of this representation is displayed in Figure 1. This choice of data structure has been guided by our desire to develop a system that can maintain all captured information while being able to rapidly respond to a great variety of queries, virtually being able to respond to requests about any number of possible time, space and semantics related criteria. The complete process by which interactions are logged by robot team members and aggregated to the data structure is shown in Figure 2.

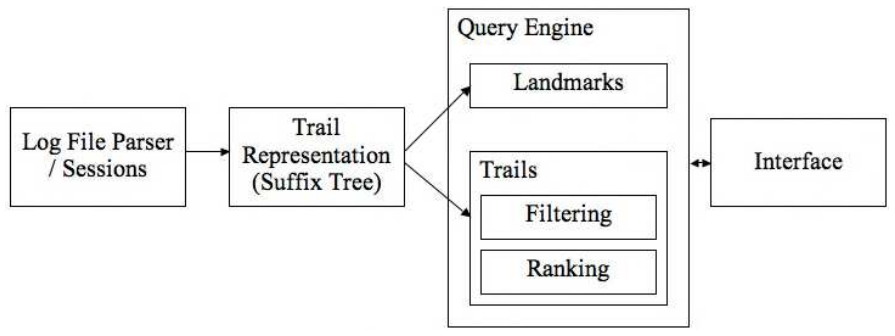

Fig. 2. Shared Memories coordination architecture.

Finally, a significant trail is a sequence of landmark visits starting and ending at a particular landmark, such that it satisfies one of more the following criteria, for example

- It is one of the top-n trails in respect of trail popularity.

- It is one of the top-n trails in respect of average time (or some other time-related statistical measure) spent interacting with the sensor nodes in the trail.

- It is one of the top-n trails in respect of relevance of the landmarks to chosen semantics (for example related to environmental temperature measurements within a spatial sub-domain).

- It is one of the top-n trails in respect of one of the above criteria for a chosen team sub-grouping.

In addition, the above criteria may be combined and weighted for example, one of the top-n trails in respect of trail popularity that last at least a specific time period. Significant trails are identified, within a particular class of trails- for example all trails that start at a specific known landmark of the particular environment navigated and end at another. Other classes can be defined by selecting for example, all trails between any two specific landmarks; all trails that start at the entrance landmark and last more than or less than a fixed period of time; all trails that pass through a specific landmark; all trails of a fixed length; all trails that start and end at the same landmark (i.e. all cyclic trails); all trails that occur within a specific time, e.g. in the morning, in the afternoon, on one or every Tuesday, or during a period with specific environmental characteristics (for instance, unusually high-temperature - with the unusual periods automatically identified by the model) to list only a few. Significant trails can be inferred from the interaction network via a variety of tree-transversal methods. We have proposed mechanisms to compute efficiently significant trails based on a fine grain heuristic defined on the interaction network, extending similar techniques developed for the web.

\section{A FEASIBILITY STUDY}

To test the feasibility of this proposal we have implemented a simple system to collect and analyze robotic trails interacting with simple wireless proximity sensors. In this case, we only analyze the aggregate collected data and we have not used it to modify the behavior of the individual robots in response to significant trails. We have observed that this approach is effective and efficient and we believe it can be easily extended to other types of wireless sensor networks.

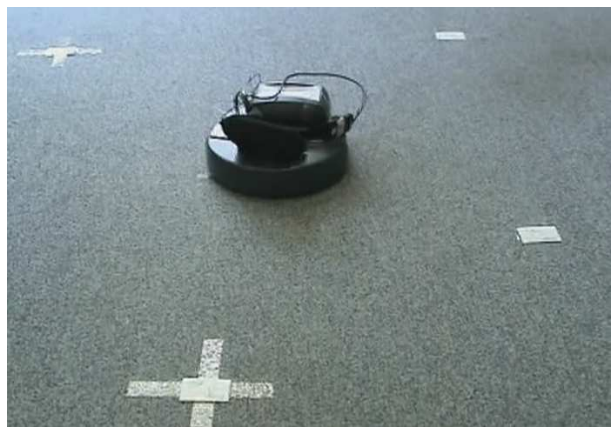

Fig. 3. iRobot Roomba with surrogate Shared Memories capture device. Also visible are the RFID tags forming a proximity detection grid on the floor.

The sensing component of our prototype is provided by simple ISO 14443 RFID proximity tags. For our robotic platform we have selected the iRobot Roomba cleaner [6] primarily due to its cost advantages. As the capabilities of the Roomba are not adequate for this experiment we have augmented it with a surrogate device based on our mobile sensor node prototype [7] instrumented with and ACG multiprotocol reader [1]. In more detail, the different components of the system play the following roles:

- Surrogate device. The Shared Memories surrogate device sits on top of the Roomba and interacts with proximity tags. Each interaction is timestamped, recorded and transmitted wirelessly to the coordination server in real-time or batch mode in case when the robot is not within range of an access point.

- Proximity sensing tags. We use proximity tags in a form of printable adhesive roll and can be stuck to or placed on almost any surface. This allows for rapid deployment of a system in virtualy any environment. 
- Coordinator. The Shared Memories coordinator runs on a server with both wired and wireless connectivity. The analysis software also provides a graphical user interface to display on the fly significant trails for observation and experimentation.

The robot, surrogate and embedded sensors is shown in Figure 3. The Shared Memories real-time statistics output is shown in Figure 4.

We have deployed this system and collected several data sets over extended system runs. Our software has been able to calculate significant trails in all cases and respond to a variety of queries under different criteria. The performance of the system overall is encouraging and we are investigating these techniques in current work.

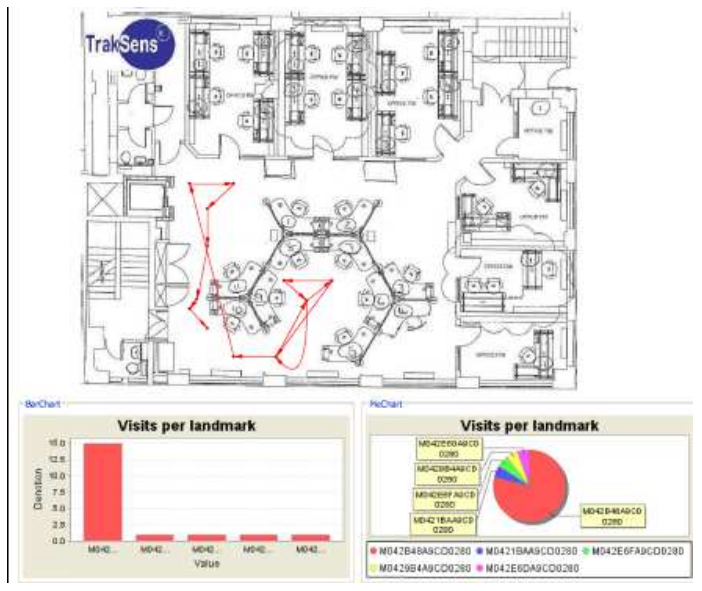

Fig. 4. Visual display of captured aggregate trails on the Shared Memories coordinator: most significant path computed, and per-landmark visitation statistics.

\section{RELATED WORK}

To be sure, the mixed use of wireless sensor networks and robotics have attracted considerable interest [3]. Nevertheless, the majority of this work is focused on two problems: the introduction of mobility within the sensor network as a means to improve robustness in the presence of node failure and increase routing performance and coverage; and the local use of the sensing elements by robots as a means to improve the information they hold about their immediate environment. To our knowledge, there are few attempts to employ the wireless sensor network as a way to coordinate teams of robotic agents by employing its ad-hoc communication capability in addition to its sensing.

Notably, rather than construct a universal model of the aggregate experience gained by a team, [8] proposes the use of embedded RFID sensor nodes as a means to store discoveries made by specific individuals to be employed as pointers by subsequent visitors to these sites. This approach has the advantage that a robot team can coordinate in a completely decentralized manner, but fails to capitalize on ample ad-hoc networking opportunities.
Within our own group, a similarly decentralized coordination algorithm has been proposed [4], with a view to provide coordination opportunities in search and rescue operations conducted by robot teams. Similar to [8], this technique uses RFID tags embedded in the environment to relay information to other team members regarding the history of operation within a particular vicinity.

\section{FUTURE WORK AND CONCLUSIONS}

In this paper, we introduce a trail-based approach to robot team coordination that makes use of ad-hoc wireless networking opportunities and the availability of wireless sensor networks embedded within the environment of operation. In particular, we have outlined the operation of the Shared Memories coordination engine which process the interaction histories of individual team members with the sensor network to construct probabilistic representations of the collective experience that can be used to provide the analysis for any query related to the team activities. Such patterns of past experience can be used to identify areas that require further attention or optimize the collective behavior of the team.

We are currently working on developing this passive coordination approach into an active coordination mechanism. In particular, we are investigating classification algorithms that allow the prediction of individual behaviors and thus provide a way to steer the team into areas that require additional attention in a manner that is both effective and efficient. From an application perspective, we are particularly interested in combining out techniques with delay-tolerant networking approaches to improve the robustness and accuracy of our trail collection performance, which is expected to have a significant effect on the capability to successfully anticipate team behaviors.

\section{REFERENCES}

[1] ACG. ACG Multi-ISO RFID Reader Manual. Available via http : //www.acg.de/

[2] J. Borges and M. Levene. Evaluating variable length Markov chain models for analysis of user web navigation sessions. IEEE Transactions on Knowledge and Data Engineering. to appear 2007.

[3] H. Chen, B-C. Cheng, C-C. Cheng, L-K. Tsai. Smart Home Sensor Networks Pose Goal-Driven Solutions to Wireless Vacuum Systems. Proc. 2006 Int. Conf. Hybrid Inf. Tech. (ICHIT’06). Vol. 2. pp. 364-373. 2006

[4] E. Ferranti, N. Trigoni and M. Levene. Brick\&Mortar: An Online MultiAgent Exploration Algorithm. IEEE Int. Conf. Robotics and Automation (ICRA). 2007.

[5] R. Golledge. Wayfinding Behavior: Cognitive Mapping and Other Spatial Processes. The Johns Hopkins University Press. 1998.

[6] T. E. Kurt. Hacking Roomba: ExtremeTech. Wiley. 2006.

[7] G. Lane, C. Brueton, D. Diall, D. Airantzis, N. Jeremijenko, G. Papamarkos, G. Roussos and K. Martin. Community-based Public Authoring with Mobile Chemical Sensor Networks. Proc. IEE Intelligent Environments. 5-6 July, Athens, Greece. 2006.

[8] M. Mamei and F. Zambonelli. Pervasive Pheromone-Based Interaction with RFID Tags. ACM Trans. Autonomous and Adaptive Systems. 2007 (in press).

[9] M. Mateas and P. Sengers. Narrative Intelligence. John Benjamins Publishing. 2003

[10] D. Papadogkonas, G. Roussos and M. Levene. Discovery and Ranking of Significant Trails. 2nd Int. Work. Expl. Context History in Smart Environments (ECHISE 2006). Irvine, CA, 17 September, 2006.

[11] P. Weiner. Linear Pattern Matching Algorithms. Proc. 14th IEEE Annual Symp. on Switching and Automata Theory. pp. 1-11. 1973. 